\title{
Analysis of biosynthetic gene clusters of Rhodococcus sp. S10
}

\author{
A. Sorokina ${ }^{1 *}$, L. Shafigullina ${ }^{1}$, A. Elistratova ${ }^{1}$, M. Belenikin $^{2}$, M. Sharipova ${ }^{1}$, \\ L. Bogomolnaya ${ }^{1,3}$, I. Khilyas ${ }^{1}$ \\ ${ }^{1}$ Institute of Fundamental Medicine and Biology KFU, Kazan, Russia \\ ${ }^{2}$ Department of Molecular and Biological Physics, Moscow Institute of Physics and Technology (State University), \\ Dolgoprudny, Moscow Region, Russia \\ ${ }^{3}$ Texas A\&M University Health Science Center, Bryan, Texas, USA \\ *e-mail: alvita.94@yandex.ru
}

Key words: siderophores, secondary metabolites, NRPS, Rhodococcus

Motivation and Aim: Bacteria produce a broad spectrum of biologically active natural compounds, including peptides, synthetized by using large multifunctional nonribosomal peptide synthetases (NRPSs) or polyketide synthases (PKSs). Among microbial peptides the compounds with a metal chelating activity draw a particular interest. Siderophores are secreted low molecular weight compounds, which can chelate Fe (III) with an extremely high affinity [1]. Bacteria from the genus Rhodococcus have been shown to produce a wide range of secondary metabolites [2]. Nowadays, the only known siderophores produced by members of the genus Rhodococcus include heterobactin A and rhodobactin [2]. The aim of this study was to identify additional biosynthetic gene clusters in the genome of Rhodococcus sp. S10.

Methods and Algorithms: Rhodococcus sp. S10 genome was analyzed for secondary metabolite and siderophore biosynthetic gene clusters using antiSmash software; RAST software was used for gene annotation [3].

Results: One hundred two biosynthetic gene clusters were predicted to be present in the genome of Rhodococcus sp. S10 by the antiSMASH software. Among those, two putative PKSs and ten putative NRPSs gene clusters were identified. Two NRPS clusters have a high sequence homology to known siderophores. Thus, cluster 99 has $100 \%$ similarity to heterobactin gene cluster of $R$. qingshengii BKS 20-40, Rhodococcus sp. ADH and $R$. erythropolis SK121; while cluster 56 has $57 \%$ similarity to albachelin gene cluster of $R$. qingshengii BKS 20-40 and $R$. erythropolis CCM2595. Five NRPS gene clusters of Rhodococcus sp. S10 did not show any homology to any known bacterial NRPS clusters. Conclusion: Analysis of Rhodococcus sp. S10 genome allowed us to identify a number of putative biosynthetic gene clusters. High homology of several Rhodococcus sp. S10 gene clusters to genes involved in siderophores synthesis encourages the search for new metabolites with metal chelating activity, which might promote Rhodococcus sp. S10 growth and adaptation to the extreme environments.

Acknowledgements: This work was performed within the Program of Competitive Growth of Kazan Federal University and supported by RFBR (grant no. 16-34-60200) and the scholarship of the President of the Russian Federation for young scientists and graduate students.

\section{References}

1. Crosa J.H., Walsh C.T. (2002) Genetics and assembly line enzymology of siderophore biosynthesis in bacteria. Microbiol. Mol. Biol. Rev. 66(2):223-249.

2. Carrano C.J. et al. (2001) Heterobactins: A new class of siderophores from Rhodococcus erythropolis IGTS8 containing both hydroxamate and catecholate donor groups. BioMetals. 14:119-125.

3. Weber T. et al. (2015) antiSMASH 3.0 - a comprehensive resource for the genome mining of biosynthetic gene clusters. Nucleic Acids Res. DOI 10.1093/nar/gkv437. 